\title{
Non-celiac wheat sensitivity: a search for the pathogenesis of a self-reported condition
}

\author{
Pasquale Mansueto, ${ }^{1}$ Maurizio Soresi, ${ }^{1}$ Rosario Iacobucci, ${ }^{1}$ Francesco La Blasca, ${ }^{1}$ Giulia Romano, ${ }^{2}$ \\ Alberto D'Alcamo, ${ }^{1}$ Antonio Carroccio ${ }^{1,3}$ \\ ${ }^{1}$ Biomedical Department of Internal Medicine and Specialties, University of Palermo; ${ }^{2}$ Internal Medicine Department, \\ Guzzardi Hospital, Vittoria (RG); ${ }^{3}$ Internal Medicine Department, Giovanni Paolo II Hospital, Sciacca (AG), Italy
}

\begin{abstract}
A significant percentage of the general population reports gastrointestinal and non-gastrointestinal symptoms caused by wheat and/or gluten ingestion, even though they do not suffer from celiac disease (CD) or wheat allergy (WS), because they test negative both for CD-specific serology and histopathology. All patients report improvement of symptoms on a gluten-free diet. This clinical condition has been named non-celiac gluten sensitivity (NCGS). The objective of this paper was to review some studies regarding the pathogenesis of NCGS to summarize the current hypotheses about the mechanisms, which can lead to NCGS. Particular attention was given to the immunologic and the malabsorptive hypotheses. We reviewed data of our previous studies involving patients diagnosed with NCWS by means of double-blind placebo-controlled wheat challenge. The data indicating a possible wheat allergy diagnosis were examined and other data in the literature were reviewed; we also reviewed the putative role of fermentable oligosaccharides disaccharides monosaccharides and polyols (FODMAPs) and of $\alpha$-amylasetrypsin inhibitors (ATIs) proteins in the NCGS pathogenesis. NCGS pathogenesis has been attributed to very different mechanisms, among others: i) activation of innate and adaptive immunity (the immune/allergic mechanisms) induced by gluten or the non-gluten ATI family; ii) incomplete digestion and/or absorption of FODMAPs (the intolerance mechanisms); and iii) psychological effect. We suggest that NCGS is a heterogeneous condition, which includes different subgroups of patients who have different pathogenic mechanisms: strong data suggest a direct pathogenic immunogenic/inflammatory role of wheat-cereal proteins (not only gluten) in a subgroup, probably the largest, of these patients.
\end{abstract}

\section{Introduction}

Celiac disease $(C D)$ prevalence is estimated to be close to 1:100 in Western countries. However, a

Correspondence: Antonio Carroccio, Biomedical Department of Internal Medicine and Specialties (Di.Bi.M.I.S), University of Palermo, 90133 Palermo, Italy.

Tel.: +39.0925.962492. E-mail: antonio.carroccio@unipa.it

Key words: Non-celiac wheat sensitivity; celiac disease; pathogenesis of non-immunoglobulin E-mediated food allergy.

Contributions: PM, AC, conceived the work; PM, FLB, AD'A, wrote the paper; RI, GR, bibliographic research; PM, MS, AC, discussed the critical points of review.

Conflict of interest: the authors declare no potential conflict of interest.

Received for publication: 19 July 2018.

Accepted for publication: 19 September 2018.

This work is licensed under a Creative Commons Attribution NonCommercial 4.0 License (CC BY-NC 4.0).

C) Copyright P. Mansueto et al., 2019

Licensee PAGEPress, Italy

Italian Journal of Medicine 2019; 13:15-23

doi:10.4081/itjm.2019.1070 much higher percentage of the general population considers itself wheat sensitive (WS), and excludes wheat from the diet on the basis of their negative experience after eating wheat-containing foods. These wheat-reactive patients often report symptoms similar to CD, but have negative CD serology and histopathology.

Recently, this new clinical condition has been framed as non-celiac gluten sensitivity (NCGS). ${ }^{1,2}$ Although it is probable that NCGS had been described as a distinct clinical condition about 40 years ago, ${ }^{3}$ it is now identified as a syndrome characterized by symptoms which can involve the gastrointestinal (GI) tract, nervous system, skin, and other organs, following the ingestion of wheat/gluten, in subjects who do not suffer from CD or wheat allergy (WA). - $^{4-6}$ The existence of this condition is controversial and specific markers are lacking.

There is little data on the pathogenesis of NCGS, and it is probable that different pathogenic mechanisms are involved in the different clinical manifestations of the disease. In this review we summarized the scientific evidence that NCGS pathogenesis involves the immune system and, in particular, we focused on the hypothesis that it could be a form of non-immunoglobulin (Ig) E-mediated food allergy. 


\section{History of the disease}

Humankind has existed in some form for about 2.5 million years, but it is only in the last 10,000 years that it has been exposed to wheat. During the $20^{\text {th }}$ century, nutritional needs increased dramatically, due to the food shortage caused by the two World Wars in the first half of the century, and the exponential growth of the world population in the second half of the century. In this context the main effort of many agronomists and geneticists was to create new wheat varieties that are stronger and richer in gluten content. In 1941, the Nutrition Society, a group of researchers interested in nutritional problems, declared the need to increase wheat production and expand the global wheat output by fivefold within the end of the $20^{\text {th }}$ century. It is possible that new kinds of wheat, particularly enriched in gluten content, have greatly contributed to the explosion of gluten-related diseases, although no data in the literature actually confirm this hypothesis. Increased wheat production (and wheat gluten content) may have had an effect on CD prevalence, which was reported in the UK in 1950 as 1:8000 inhabitants and has increased to about $1 \%$ of the general population in recent decades. In addition, the discovery in recent decades of more and more accurate serology assays for $\mathrm{CD}$ diagnosis has simplified the diagnostic work-up, contributing to the increase in reported $\mathrm{CD}$ prevalence. ${ }^{7-10}$

The recent rise of the market for gluten-free products in Europe and in the United States was also influenced, in part, by advertising campaigns that claim a medical need for a gluten-free diet (GFD), which exceeds the foreseeable consumption of the CD patient population. This is just one of the factors that raises questions about possible gluten reactions apart from CD and WA. ${ }^{11,12}$ The non-medical press has suggested that 17 million Americans are gluten sensitive. It must be remembered that this is a big business, with a projected increase in the market for gluten-free products from $\$ 100$ million in 2003 , to exceeding $\$ 10$ billion in the year 2015 , and projected to $\$ 24$ billion by the year $2020 .{ }^{13}$

The objective of this paper was to review some studies regarding the pathogenesis of NCGS to summarize the current hypotheses about the mechanisms, which can lead to NCGS. Particular attention was given to the immunologic and the malabsorptive hypotheses.

\section{Self-reported non-celiac gluten sensitivity - the size of the problem}

The NCGS diagnosis is often suggested by the patients themselves, who report GI and extra-intestinal symptoms after wheat/gluten ingestion and become asymptomatic on GFD. However, the lack of diagnostic biomarkers for NCGS is an unsolved problem; consequently, the real frequency of NCGS itself is controversial. Previous works, based on administering questionnaires, have reported a wide range of self-reported NCGS prevalence world-wide, between $0.6 \%$ and $13 \%$, with lower percentages of adherence to a GFD. ${ }^{14-19}$

Recently, the first similar study performed in two high schools in the south of Sicily (Italy), on a total of 555 students, ${ }^{20}$ showed that self-reported NCGS is also common in teenagers. In particular, we demonstrated that among these students (mean age 17 years), $1.26 \%$ had an established diagnosis of $\mathrm{CD}$, whereas the prevalence of self-reported NCGS was $12.2 \%$. In the whole group, $2.9 \%$ of non-celiac subjects were consuming a GFD. Very often, the self-reported NCGS patients had irritable bowel syndrome (IBS) symptoms (44\%), confirming an overlap between these clinical conditions. The same study underlined one of the most important problems of the new NCGS era: only $23 \%$ of NCGS self-reporters had consulted a doctor about this problem, and only $14 \%$ had undergone serology tests for CD. Thus, in the great majority of the cases NCGS remained a self-diagnosis, without any medical evaluation and a probability that a CD diagnosis was missed due to the lack of clinical investigations. In any case, the strong similarity between the percentages of self-reported NCGS and self-prescribed GFD, observed both in teenagers, and in adults previously reported, seems to suggest that self-reported NCGS is a problem perceived in all age groups.

\section{Pathogenetic mechanisms of non-celiac gluten sensitivity}

Despite the great interest in NCGS, much remains unknown about the pathogenesis. Some studies suggest that wheat components other than gluten can cause the symptoms. NCGS pathogenesis has been attributed to very different mechanisms, among others: i) activation of innate and adaptive immunity (the immune/allergic mechanisms) induced by gluten or the non-gluten $\alpha$ amylase/trypsin inhibitor (ATI) family; ii) incomplete digestion and/or absorption of fermentable oligosaccharides and disaccharides, monosaccharides and polyols (FODMAPs) (the intolerance mechanisms); and iii) psychological effect. ${ }^{21-25}$

\section{The allergy hypothesis}

In the pathogenic context of non-IgE-mediated allergic mechanisms, the following have been reported: association of NCGS with other food hypersensitivities, nickel allergy, together with 
structural/functional immunologic/inflammatory changes in the intestinal mucosa after food challenge, with systemic immune activation data, linked to intestinal epithelium damage, and autoimmune disease and auto-antibodies. ${ }^{26}$

\section{Association with other food hypersensitivities}

In 2012, we reviewed the clinical charts of all subjects with an IBS-like presentation who had been diagnosed with NCGS using a double-blind placebocontrolled challenge in the years $2001-2011 .{ }^{27} \mathrm{CD}$ and IBS patients served as controls. Our results demonstrated that NCGS is a heterogeneous clinical condition, comprising various subgroups of patients who have different clinical histories and characteristics. We identified at least two distinct kinds of NCGS: one group of patients with NCGS alone, and another of subjects who showed clinical symptoms when exposed to wheat, cow's milk protein, and many other foods. These two groups clearly varied in presentation and immunological findings, although there was also a significant overlap. Subjects with NCGS alone, although having negative CD serology and normal duodenal villi histology, had a higher frequency of family history of CD, the DQ2 or DQ8 HLA haplotype, duodenal lymphocytosis and positive EmA assay in the culture medium of the duodenal biopsies. On this basis we suggested that it is possible that a percentage of these patients $(C D$-like) could be predisposed to develop villous atrophy - overt $\mathrm{CD}$ - in the future. The NCGS patients having multiple food hypersensitivity presented characteristics more similar to those of allergic rather than CD patients, although none tested positive on IgE-based assays. In accordance with the allergy hypothesis, these patients (Allergy-like) showed a higher frequency of family and personal history of food allergy in infancy and coexistent atopy than the other group. Their predominance in our study probably conditioned the results of the immunology assays (positive for serum anti-gliadin and anti- $\beta$-lactoglobulin antibodies of $\operatorname{IgG}$ class and basophil activation assay), and the histology studies (mucosal eosinophil infiltration in the duodenum and colon). Regarding the frequency of positive serum anti-gliadin antibodies in NCGS, other studies demonstrated that $56 \%$ and $8 \%$ of NCGS patients were positive for IgG and $\operatorname{IgA}$ anti-gliadin antibodies, respectively, ${ }^{28}$ and that anti-gliadin antibodies of the IgG class disappear in patients with NCGS, reflecting a strict compliance to the GFD and a good clinical response to gluten withdrawal. ${ }^{29}$

\section{Association with nickel allergy}

Another study supporting a possible pathogenic association between NCGS and allergy evaluated the frequency of contact dermatitis due to nickel allergy in NCGS patients diagnosed by a double-blind placebo-controlled challenge. ${ }^{30}$ It was a prospective study of NCGS patients, with age- and sex-matched subjects with functional GI symptoms who served as controls. In these study groups, $10 \%$ patients with NCGS suffered from contact dermatitis and nickel allergy and this frequency was statistically higher than that observed in the control group. The main clinical characteristic of NCGS patients with nickel allergy was a higher frequency of cutaneous symptoms after wheat ingestion compared to NCGS patients who did not suffer from nickel allergy. Apart from the association, this study suggested that nickel allergy should be evaluated in NCGS patients who have cutaneous manifestations after wheat ingestion. Furthermore, it is interesting to recall that some immunologic pathways involved in the nickelinduced mucositis and dermatitis, i.e. the inflammatory response via the activation of TLR4 and the infiltration of lymphocytes that secrete interferon-gamma (IFN)- $\gamma,{ }^{31,32}$ have also been hypothesized or demonstrated in NCGS. ${ }^{33-37}$

In this context, i.e. the link between NCGS and allergy, including nickel allergy, a previous Italian multicenter study of about 500 patients is noteworthy, finding that more than $20 \%$ of suspected NCGS patients had an allergy to one or more inhalants, food, or metals, the most frequent being mites (26\%), gramineae (24\%), nickel (15\%), cat/dog hair (13\%), shellfish ( $8 \%)$, and parietaria pollen $(6 \%){ }^{38}$

Another study ${ }^{39}$ of allergic patients with the classical symptoms, such as asthma, rhinitis or contact dermatitis, and also GI symptoms (diarrhea, abdominal pain, and weight loss) of obscure origin, showed that 77 of 262 patients $(29 \%)$ exhibited histological damage of the duodenal intestinal mucosa. These subjects tested positive to prick test versus aeroallergens, such as pollen and Dermatophagoides (D.) pteronyssinus and/or $D$. farinae, and/or positive to patch test versus contact allergens (mainly nickel and cobalt, and at a lower rate to thimerosal, fragrance mix, balsam of Peru and potassium dichromate). The patients with duodenal mucosa inflammation were asked to follow a GFD despite not reporting wheat intolerance. On this diet they exhibited control of symptoms as well as stabilization of the hematological parameters (i.e. anemia of inflammatory origin), even though allergic manifestations did not abate. It is noteworthy that all these patients were negative to the anti-gliadin and anti-endomysium antibodies and did not carry the DQ2 and DQ8 haplotypes. The authors recommended that biopsy should be part of the routine investigation of allergic disease to offer the benefits of treatment with a GFD to the patients. 


\section{Association with structural/functional immunologic/inflammatory changes in the intestinal mucosa after food challenge}

The relationship between food ingestion and gastro-intestinal motility disorders, including the NCGS clinical aspects, is still unclear but clinically evident, and has received paramount input by the endoscopy study of Fritscher-Ravens and coll. ${ }^{40}$ They investigated suspected food intolerance in patients with IBS using confocal laser endomicroscopy (CLE) for real-time visualization of structural/functional changes in the intestinal mucosa after food challenge. Patients with endoscopic changes after food challenge (CLE + ) were put on personalized exclusion diets and followed up for long-term symptom relief. Diluted food antigens were administered directly to the duodenal mucosa through the working channel of the endoscope. Epithelial breaks, intervillous spaces, and the number of intraepithelial lymphocytes (IELs) were measured before and after the food challenge. CLE showed a real-time response to food antigens in the majority of patients (22 of 36). Specifically, 13 patients reacted to wheat, 9 to milk, 6 to yeast and 4 to soy. Baseline IELs were significantly higher in CLE+ than CLE- subjects. Within 5 minutes of exposure of CLE + patients to food antigens, epithelial leaks/gaps formed, intervillous spaces widened, and IELs increased. IBS symptom scores improved more than 50\% in CLE+ patients after a 4-week exclusion diet and increased to $74 \%$ at 12 months; symptoms continued in CLE- patients. The Authors concluded that CLE is an excellent instrument to demonstrate food-related reactions in IBS. We add that CLE could be an excellent method to identify a subgroup of the NCGS patients - those with non-IgE-mediated WA from the confusing melting pot that NCGS still is. It is very interesting that in this study the second most frequently offending food, after wheat, was cow's milk; this frequent association between wheat and cow's milk intolerance/allergy in IBS patients is identical to the result of our previous clinical study. ${ }^{27}$

\section{Association with systemic immune activation data, linked to intestinal epithelium damage}

A recent study by Uhde and coll. has contributed data on the possible mechanisms that underline the intestinal epithelium breakdown after food (wheat) ingestion/contact in NCGS patients. ${ }^{41}$ This study aimed to determine if NCGS is associated with systemic immune activation that may be linked to an enteropathy (i.e. intestinal epithelial barrier defects), leading to an inadequate regulation of the interaction between the gut microbiota and systemic circulation, resulting in peripheral immune activation. To examine this the authors measured, in self-reported NCGS patients, with $\mathrm{CD}$ and healthy subjects as controls, the levels of lipopolysaccharide (LPS)-binding protein (LBP) and soluble CD14 (sCD14), as indicators of the translocation of microbial products, particularly LPS, across the epithelial barrier. Translocated circulating LPS can result in the rapid secretion of LBP by GI and hepatic epithelial cells, as well as sCD14 by CD14+ monocytes/macrophages. The sCD14 binds LPS in the presence of LBP to activate TLR4. The authors demonstrated significantly elevated serum levels of both LBP and sCD14 in individuals with NCGS compared to patients with $\mathrm{CD}$ and healthy controls. There was a high degree of correlation between serum LBP and sCD14, suggesting that these molecules are concurrently expressed in response to the stimulus in NCGS individuals. They also quantified serum levels of antibody to LPS core oligosaccharide, or EndoCAb, which is known to modulate in response to bacterial endotoxin in circulation. The NCGS cohort exhibited increased levels of EndoCAb IgM. Systemic immune response in self-reported NCGS patients was not limited to only LPS, as demonstrated by higher levels of $\operatorname{IgG}$ and $\operatorname{IgM}$ antibodies also to flagellin in comparison with $\mathrm{CD}$ patients and healthy controls. Considering that no individuals in this study had evidence of infection, these observations suggest a translocation of microbial products from the GI tract that contributes to the observed innate and adaptive immune activation in the NCGS cohort. Finally, they considered whether the observed systemic immune activation in response to microbial products in individuals with NCGS might be linked to increased intestinal enterocyte damage. Fatty acid-binding protein 2 (FABP2) concentrations, a cytosolic protein specific to intestinal epithelial cells, that is rapidly released into systemic circulation after cellular damage, in the NCGS cohort correlated strongly with levels of LBP and SCD14, suggesting a link between the intestinal epithelial cell damage and the acute systemic immune activation in response to translocated microbial products. Furthermore, there was a significant change towards normalization of the levels of FABP2 and immune activation markers in a subgroup of individuals with self-reported NCGS who consumed a diet excluding wheat and related cereals, thus suggesting a wheat-dependent state of systemic immune activation in conjunction with a compromised intestinal epithelium affecting self-reported NCGS.

One of the next relevant steps in the study of NCGS pathogenesis is to understand the respective role of innate and adaptive immunity. The allergy hypothesis clearly points to the determining role of adaptive immunity, but there were interesting data indicating a possible role for an activation of innate immunity pathways in NCGS patients. The first studies on NCGS pathogenesis showed that the 
expression of the innate immunity marker Toll-like receptor 2 was higher in NCGS but not in celiac patients and suggested that NCGS was a condition associated with prevalent gluten-induced activation of innate, rather than adaptive, immune responses. ${ }^{42}$

More recently, our group focused on the immunologic response of the rectal mucosa in NCGS patients. ${ }^{43}$ The prospective study included patients with IBS-like clinical presentation, diagnosed with NCGS by double-blind placebo-controlled challenge, and IBS patients not improving on wheat-free diet, used as controls. The rectal biopsies from wheat-challenged NCGS patients showed a significant mucosal CD45+ infiltrate, consisted of CD3+ and CD3- lymphocytes, with the latter spontaneously producing more IFN- $\gamma$ than IBS controls. About $30 \%$ of IFN- $\gamma$-producing CD45+ cells were T-bet+, CD56-, natural killer (NK) P44-, and CD117-, defining them as a type-1 innate lymphoid cells (ILC1). We recall that ILCs are divided into 3 major groups on the basis of a different transcriptional control and cytokine responsiveness and production. Group 1 ILCs include both NK and noncytolytic ILC1. These non-cytolytic ILC1 are characterized by the expression of T-bet and the production of IFN- $\gamma$ in response to interleukin (IL)-12, IL-15, and IL-18 and thus resemble CD4+ T helper 1 cell innate counterparts. In our study, Group 1 IFN- $\gamma-$ producing ILC1 cells significantly decreased in patients analyzed two weeks after they resumed a wheat-free diet. These data indicate that in patients with active NCGS, IFN- $\gamma$-producing ILC1 cells infiltrate rectal mucosa and support a role for this innate lymphoid cell population in the pathogenesis of NCGS.

\section{Association with autoimmune disease and auto-antibodies}

Other clinical and laboratory data are relevant when we consider the relationship between the NCGS clinical condition and the altered immune regulatory function. A few years ago, in a study that unified a retrospective and a prospective analysis, ${ }^{44}$ we evaluated the prevalence of autoimmune diseases (ADs) among patients with NCGS, and investigated whether or not they carry serum antinuclear antibodies (ANA). CD and IBS patients served as controls. In the retrospective analysis, similar portions of NCGS $(29 \%)$ and CD (29\%) patients had ADs (mainly Hashimoto's thyroiditis), compared with a smaller proportion of subjects with IBS (4\%). In the prospective study, $24 \%$ of NCGS, $20 \%$ of CD, and $2 \%$ of IBS patients had ADs. In the retrospective study, serum samples tested positive for ANA in $46 \%$ of NCGS (median titer, $1: 80$ ), in $24 \%$ of CD, and in $2 \%$ of IBS patients; in the prospective study, serum samples were positive for ANA in $28 \%$ of NCGS, $7.5 \%$ of $\mathrm{CD}$, and $6 \%$ of IBS patients. ANA positives were associated with the presence of the HLA DQ2/DQ8 haplotypes.

\section{Immune system activation: a role for $\alpha$-amylase/trypsin inhibitor?}

Wheat proteins can be classified into albumins, globulins, gliadins, and glutenins, according to their structural properties and solubility. Commercial wheat contains approximately 10\%-20\% albumins/globulins and $80 \%-90 \%$ gluten, a complex mixture of monomeric gliadins and polymeric glutenins that are partly resistant to GI enzymatic proteolysis. Albumins and globulins constitute up to 14 families of proteins, such as the ATIs, $\beta$-amylases, peroxidases, lipid transfer proteins, serpins, and others. ATIs are present in many plants, including wheat and other cereals, where they may be involved in grain maturation and carbohydrate storage, but they also inhibit enzymes of common parasites, such as mealworms and mealbugs. ${ }^{45}$

Wheat ATIs represent between 2 and $4 \%$ of total wheat protein and are strong inducers of innate immune responses. Specifically, ATI family members activate the TLR4-MD2-CD14 complex, resulting in activation of both the classic (nuclear factor kappa B) and the nonclassic (interferon responsive factor 3) pathway, leading to up-regulation of dendrite cell maturation markers and release of pro-inflammatory innate cytokines (i.e. TNF$\alpha$, IL-8 and MCP-1 [CCL-2]) from activated myeloid cells, thus eliciting strong innate immune effects, not only in vitro but also in vivo after oral or systemic challenge, mostly in the colon, then in the ileum, and then in the duodenum. Morphological effects of ATIs in the gut are discrete and may reflect dendrite cell or macrophage activation rather than mirror an increase in inflammatory cell numbers, which is well in line with the histology previously described in patients with NCGS. This might have important implications not only for the onset and severity of inflammatory intestinal diseases, but also as a likely contributor to NCGS pathogenesis. ${ }^{46-48}$ Therefore, since it is possible that non-gluten components of wheat, barley and rye induce the disease, we prefer the term non-celiac (nonallergy) wheat sensitivity (NCWS), at least in scientific discussions. ${ }^{49,50}$

\section{Non-immunologic pathogenesis of non-celiac gluten sensitivity: the role of fermentable oligosaccharides, disaccharides, monosaccharides, and polyols}

Throughout the 1980s and 1990s, evidence (especially observational cohort studies) of a possible induction of functional bowel disorders and IBS symptoms due to the poorly absorbed, short-chain carbohydrates and polyols (lactose, fructose, and sorbitol) has confirmed that dietary restriction of all 
three together could cause symptomatic relief. More recent studies confirmed the above-mentioned results. However, it is clear that these sugars are not the only answer. A deeper examination of the international literature on the biochemistry and physiology of digestion of other carbohydrates suggests involvement of fructo-oligosaccharides (fructans or FOS) and galacto-oligosaccharides (galactans or GOS); they are also short-chain carbohydrates and are incompletely absorbed in the human GI tract. Other potential culprits seem to be incompletely absorbed polyols (i.e. mannitol, maltitol, and xylitol), used as artificial sweeteners, but also found naturally in foods. Grouping these poorly absorbed, short-chain carbohydrates according to their chain length resulted in the acronym FODMAPs (fermentable oligo-, di-, and monosaccharides and polyols). In 2005 this term was specifically coined by a group of Australian researchers who hypothesized that foods containing these forms of carbohydrates worsen the symptoms of some digestive disorders, such as IBS and inflammatory bowel disease. ${ }^{51}$ These short-chain highly osmotic carbohydrates are poorly absorbed in the small intestine and are rapidly fermented by bacteria in the gut, causing increased gas production, bowel distension, bloating, cramping, and diarrhea all symptoms of IBS, triggered in association with intrinsic visceral hypersensitivity.

In the context of the FODMAP hypothesis (i.e. nonimmunologic/non-allergic pathogenesis, or intolerance) of NCGS, Biesiekierski and coll., in a placebocontrolled, crossover re-challenge study, ${ }^{52}$ did not find any evidence of specific effects of gluten in patients with NCGS put on diets low in FODMAPs. Consequently, the authors suggested that NCGS might not be a real entity and that it might be confounded by FODMAP restriction. Accordingly, they concluded that at least in their highly selected cohort, gluten might not be a specific trigger of functional gut symptoms once dietary FODMAPs are reduced. This point of view considers the toxic effect of gluten in patients with NCGS, previously demonstrated in several other studies and by these authors themselves, ${ }^{53}$ only as a contribution to the FODMAP dietary load.

More recently, Skodje and coll..$^{54}$ performed a double-blind crossover challenge of individuals on a self-initiated GFD, for whom CD had been excluded. Participants were randomly assigned to groups put on diets containing gluten, fructans, or placebo, for 7 days. Following a minimum 7-day washout period (until the symptoms induced by the previous challenge were resolved), participants crossed over into a different group, until they completed all 3 challenges (gluten, fructan, and placebo). Symptoms were measured by the GI symptom rating scale, irritable bowel syndrome (GSRS-IBS) version. No significant effect of gluten was found compared with fructan and placebo. In contrast, a small daily dose of fructans induced greater symptoms on multiple criteria, including the overall GSRS-IBS, after a 7-day challenge. On group level, the difference compared to placebo was significantly higher after fructan challenge than after gluten challenge. Thirteen participants had their highest symptom score after gluten, while 27 had their lowest score after gluten challenge. Fructan and placebo challenge induced the highest score in 24 and 22 participants, respectively.

We would underline, however, that the inclusion criteria might have determined the different, sometimes opposite, results of the different studies on NCGS. In the study by Biesiekirski ${ }^{52}$ the characteristics of the patients included were very different from those of the patients included in our and other studies. The majority of patients that we studied (previous and current studies), exhibited intraepithelial inflammation in the duodenal mucosa (Marsh 1 lesion), and fifty percent of them carried the DQ2 HLA antigens, ${ }^{27,43}$ but both of these criteria were considered exclusionary in the Biesiekierski study. Thus, two different patient populations with NCGS were studied so the results cannot be compared. Since mucosal inflammation is a characteristic of intestinal allergic diseases, and Marsh 1 lesion can be present in several conditions other than celiac disease, we would suggest not excluding patients with duodenal lymphocytosis from future studies.

Regarding the confounding role of the low FODMAP diet in the context of NCGS, it is useful to focus on the study of McIntosh and coll. concerning the effects of a low or high FODMAP diet in patients suffering from IBS..$^{55}$ This study confirmed that the IBS symptom severity score improves on a low FODMAP diet, and showed the metabolic profile of urine in IBS patients on a low or high FODMAP diet. There was an eight-fold reduction in urinary histamine in the low FODMAP group. Obviously, this reduction could be a key to the effectiveness of the low FODMAP diet, since the histamine levels could be a measure of the immune activation in the intestinal mucosa of IBS patients. Thus, the question is What in the low FODMAP diet reduces the immune activation? Unfortunately, the Authors of that paper did not specify the composition of the diets administered to the study groups. It would be useful to know that, but both wheat and milk are commonly excluded in a low FODMAP diet.

Consequently, the evidence of the effectiveness of a low FODMAP diet does not contradict the evidence of a wheat-free diet in patients with IBS symptoms who are labeled as NCGS. It is clear that, among the possible offending FODMAPs, if wheat, milk, or other foods are identified as the specific culprits in 
determining IBS symptoms in specific patients, we would suggest a less restrictive diet and this could also mitigate the negative impact on the microbiota, which characterizes the low FODMAP diet. ${ }^{56,57}$

In summary, regarding the role of FODMAPs in

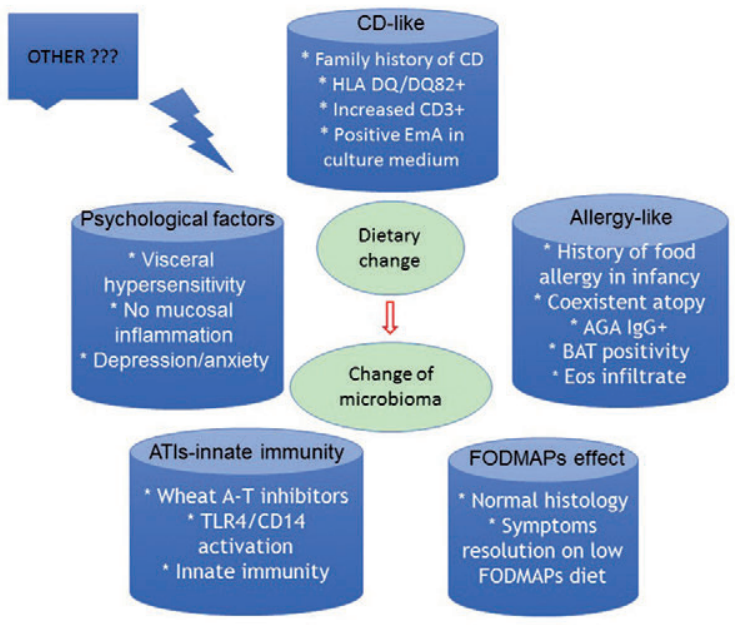

Figure 1. Different subgroups of patients suffering from non-celiac gluten/wheat sensitivity (NCGS) can recognize different pathogenesis.

The hypothesis that NCGS could be a very early phase of celiac disease (CD) should be considered; this subgroup of patients could be identified by the positivity of antiendomysial assay in the culture medium of the mucosal biopsy or by the t-TG deposits in the duodenal mucosa.

The hypothesis of a non-IgE-mediated wheat-allergy could be considered in the subjects who have coexistent atopic disease and/or a history of food allergy in infancy. Suggestive laboratory data can be serum anti-gliadin antibodies, or in vitro basophil activation test positivity. Eosinophils mucosal infiltration both in the duodenum and in rectum could be another clue.

The fermentable oligosaccharides, disaccharides, monosaccharides, and polyols (FODMAPs) hypothesis should be primarily considered in patients who did not carry the HLA DQ2 or DQ8 antigens, who had a completely normal intestinal histology and no history of coexistent atopy.

The $\alpha$-amylase/trypsin inhibitor (ATI) hypothesis is very promising and intriguing. The pathogenesis is likely linked to activation of the intestinal and extraintestinal innate immune system (monocytes, macrophages, dendritic cells) by nutritional ATIs from wheat, rye, or barley.

The hypothesis of psychological factors in the pathogenesis of NCGS is supported by the clinical overlap with the irritable bowel syndrome and by the frequent nocebo effect observed in the studies performed with the double-blind placebo-controlled method.

Other, still unknown, factors could play a role in the NCGS pathogenesis. Among these, a change in the intestinal microbiota, induced by the dietary habit change (the exclusion diet - wheat-free diet) could be a pivotal event.
NCGS, we think that the literature data support the hypothesis that a low FODMAP diet can be useful in a percentage of these patients, probably those in whom a biochemical-digestive mechanism plays a prevalent role in causing the symptoms. However, as we have suggested, we think that NCGS is a heterogeneous condition, which includes different subgroups of patients who have different pathogenic mechanisms: strong data suggest a direct pathogenic immunogenic/ inflammatory role of wheat-cereal proteins (not only gluten) in a subgroup, probably the largest, of these patients (Figure 1). ${ }^{58}$

\section{Conclusions}

There are strong data to suggest that NCGS has an immune-mediated pathogenesis and that it could be considered, for some percentage of the cases, a nonIgE-mediated, allergic-like condition. However, since the clinical presentation is heterogeneous and a serology marker is still lacking, the current group of people who avoid gluten is a melting pot, which includes patients with different pathogenesis. The real percentage of NCGS in this melting pot remains to be established, but our experience suggests that one third of IBS patients could benefit from a wheat-free diet, or a more restricted elimination diet. If confirmed, it would be a revolutionary approach to the functional gastro-intestinal disorders.

\section{References}

1. Mansueto P, Seidita A, D'Alcamo A, Carroccio A. Nonceliac gluten sensitivity: literature review. J Am Coll Nutr 2014;33:39-54.

2. Mansueto P, D'Alcamo A, Seidita A, Carroccio A. Food allergy in irritable bowel syndrome: the case of nonceliac wheat sensitivity. World J Gastroenterol 2015;21: 7089-109.

3. Ellis A, Linaker BD. Non celiac gluten sensitivity. Lancet 1978;1:1386-9.

4. Catassi C, Elli L, Bonaz B, Bouma G, et al. Diagnosis of non-celiac gluten sensitivity (NCGS): the Salerno experts' criteria. Nutrients 2015;7:4966-77.

5. Elli L, Villalta D, Roncoroni L, et al. Nomenclature and diagnosis of gluten-related disorders: A position statement by the Italian Association of Hospital Gastroenterologists and Endoscopists (AIGO). Dig Liver Dis 2017;49:138-46.

6. Volta U, Caio G, Karunaratne TB, et al. Non-coeliac gluten/wheat sensitivity: advances in knowledge and relevant questions. Expert Rev Gastroenterol Hepatol 2017;11:9-18.

7. Avidson LS, Fountain JR. Incidence of the sprue syndrome; with some observations on the natural history. Br Med J 1950;1:1157-61.

8. Fasano A, Berti I, Gerarduzzi T, et al. Prevalence of celiac disease in at-risk and not-at-risk groups in the 
United States: a large multicenter study. Arch Intern Med 2003;163:286-92.

9. Catassi C, Gatti S, Fasano A. The new epidemiology of celiac disease. J Pediatr Gastroenterol Nutr 2014;59:S7-9.

10. Ludvigsson JF, Rubio-Tapia A, van Dyke CT, et al. Increasing incidence of celiac disease in a North American population. Am J Gastroenterol 2013;108:818-24.

11. Di Giacomo DV, Tennyson CA, Green PH, Demmer RT. Prevalence of gluten-free diet adherence among individuals without celiac disease in the USA: results from the Continuous National Health and Nutrition Examination Survey 2009-2010. Scand J Gastroenterol 2013;48:921-5.

12. Newberry C, McKnight L, Sarav M, Pickett-Blakely O. Going gluten free: the history and nutritional implications of today's most popular diet. Curr Gastroenterol Rep 2017;19:54.

13 Statista. Gluten-free and free-from food retail sales in the United States from 2006 to 2020 (in billion U.S. dollars); 2013. Available from: https://www.statista. com/sta- tistics/261099/us-gluten-free-and-free-fromretail-sales/ Accessed: 4 April 2018.

14. Vasagar B, Cox J, Herion JT, Ivanoff E. World epidemiology of non-celiac gluten sensitivity. Minerva Gastroenterol Dietol 2017;63:5-15.

15. Lionetti E, Pulvirenti A, Vallorani M, et al. Re-challenge studies in non-celiac gluten sensitivity: a systematic review and meta-analysis. Front Physiol 2017;8:621.

16. Van Gils T, Nijeboer P, Ijssennagger CE, et al. Prevalence and characterization of self-reported gluten sensitivity in the Netherlands. Nutrients 2016;8:pii:E714.

17. Ramírez-Cervantes KL, Romero-López AV, NúñezÁlvarez CA, et al. Adherence to a gluten-free diet in Mexican subjects with gluten-related disorders: a high prevalence of inadvertent gluten intake. Rev Invest Clin 2016;68:229-34.

18. Ontiveros N, Rodríguez-Bellegarrigue CI, GaliciaRodríguez G, et al. Prevalence of self-reported gluten-related disorders and adherence to a gluten-free diet in Salvadoran adult population. Int J Environ Res Public Health 2018;15:pii:E786.

19. Cabrera-Chávez F, Dezar GV, Islas-Zamorano AP, et al. Prevalence of self-reported gluten sensitivity and adherence to a gluten-free diet in Argentinian adult population. Nutrients 2017;9:pii:E81.

20. Carroccio A, Giambalvo O, Blasca F, et al. Self-reported non-celiac wheat sensitivity in high school students: demographic and clinical characteristics. Nutrients 2017;9:pii:E771.

21. Volta U, Caio G, Tovoli F, De Giorgio R. Non-celiac gluten sensitivity: questions still to be answered despite increasing awareness. Cell Mol Immunol 2013;10:383-92.

22. Volta U, Caio G, De Giorgio R, et al. Non-celiac gluten sensitivity: a work-in-progress entity in the spectrum of wheat-related disorders. Best Pract Res Clin Gastroenterol 2015;29:477-91.

23. Lebwohl B, Ludvigsson JF, Green PH. Celiac disease and non-celiac gluten sensitivity. BMJ 2015;351:h4347.

24. Caio G, Riegler G, Patturelli M, et al. Pathophysiology of non-celiac gluten sensitivity: where are we now? Minerva Gastroenterol Dietol 2017;63:16-21.

25. Rotondi Aufiero V, Fasano A, Mazzarella G. Non-celiac gluten sensitivity: how its gut immune activation and potential dietary management differ from celiac disease. Mol Nutr Food Res 2018;62:e1700854.

26. Carroccio A, Mansueto P, D'Alcamo A, Iacono G. Nonceliac wheat sensitivity as an allergic condition: personal experience and narrative review. Am J Gastroenterol 2013;108:1845-52.

27. Carroccio A, Mansueto P, Iacono G, et al. Non-celiac wheat sensitivity diagnosed by double-blind placebocontrolled challenge: exploring a new clinical entity. Am J Gastroenterol 2012;107:1898-906.

28. Volta U, Tovoli F, Cicola R, et al. Serological tests in gluten sensitivity (nonceliac gluten intolerance). J Clin Gastroenterol 2012;46:680-5.

29. Caio G, Volta U, Tovoli F, De Giorgio R. Effect of gluten free diet on immune response to gliadin in patients with non-celiac gluten sensitivity. BMC Gastroenterol 2014; $14: 26$.

30. D'Alcamo A, Mansueto P, Soresi M, et al. Contact dermatitis due to nickel allergy in patients suffering from non-celiac wheat sensitivity. Nutrients 2017;9:pii:E103.

31. Di Tola M, Marino M, Amodeo R, et al. Immunological characterization of the allergic contact mucositis related to the ingestion of nickel-rich foods. Immunobiology 2014;219:522-30.

32. Dyring-Andersen B, Skov L, Løvendorf MB, et al. CD4(+) T cells producing interleukin (IL)-17, IL-22 and interferon $-\gamma$ are major effector T cells in nickel allergy. Contact Dermatitis 2013;68:339-47.

33. Sapone A, Lammers KM, Mazzarella G, et al. Differential mucosal IL-17 expression in two gliadininduced disorders: gluten sensitivity and the autoimmune enteropathy celiac disease. Int Arch Allergy Immunol 2010;152:75-80.

34. Brottveit M, Beitnes AC, Tollefsen S, et al. Mucosal cytokine response after short-term gluten challenge in celiac disease and non-celiac gluten sensitivity. Am J Gastroenterol 2013;108:842-50.

35. Vazquez-Roque MI, Camilleri M, Smyrk T, et al. A controller trial of gluten-free diet in patients with irritable bowel syndrome-diarrhea: effects on bowel frequency and intestinal function. Gastroenterology 2013;144:903-11.

36. Di Sabatino A, Giuffrida P, Fornasa G, et al. Innate and adaptive immunity in self-reported nonceliac gluten sensitivity versus celiac disease. Dig Liver Dis 2016;48: 745-52.

37. Junker Y, Zeissig S, Kim SJ, et al. Wheat amylase trypsin inhibitors drive intestinal inflammation via activation of toll-like receptor 4. J Exp Med 2012;209: 2395-408.

38. Volta U, Bardella MT, Calabrò A, et al. Study Group for Non-Celiac Gluten Sensitivity. An Italian prospective multicenter survey on patients suspected of having nonceliac gluten sensitivity. BMC Med 2014;12:85.

39. Massari S, Liso M, De Santis L, et al. Occurrence of nonceliac gluten sensitivity in patients with allergic disease. Int Arch Allergy Immunol 2011;155:389-94.

40. Fritscher-Ravens A, Schuppan D, Ellrichmann M, et al. Confocal endomicroscopy shows food-associated changes in the intestinal mucosa of patients with irritable bowel syndrome. Gastroenterology 2014;147:1012-20.

41. Uhde M, Ajamian M, Caio G, et al. Intestinal cell damage and systemic immune activation in individuals 
reporting sensitivity to wheat in the absence of coeliac disease. Gut 2016;65:1930-7.

42. Sapone A, Lammers KM, Casolaro V, et al. Divergence of gut permeability and mucosal immune gene expression in two gluten-associated conditions: celiac disease and gluten sensitivity. BMC Med 2011;9:23.

43. Di Liberto D, Mansueto P, D'Alcamo A, et al. Predominance of type 1 innate lymphoid cells in the rectal mucosa of patients with non-celiac wheat sensitivity: reversal after a wheat-free diet. Clin Transl Gastroenterol 2016;7:e178.

44. Carroccio A, D'Alcamo A, Cavataio F et al. High proportions of people with nonceliac wheat sensitivity have autoimmune disease or antinuclear antibodies. Gastroenterology 2015;149:596-603.

45. Mills EN, Jenkins JA, Alcocer MJ, Shewry PR. Structural, biological, and evolutionary relationships of plant food allergens sensitizing via the gastrointestinal tract. Crit Rev Food Sci Nutr 2004;44:379-407.

46. Zevallos VF, Raker V, Tenzer S, et al. Nutritional wheat amylase-trypsin inhibitors promote intestinal inflammation via activation of myeloid cells. Gastroenterology 2017;152:1100-13.

47. Schuppan D, Zevallos V. Wheat amylase trypsin inhibitors as nutritional activators of innate immunity. Dig Dis 2015;33:260-3.

48. Schuppan D, Pickert G, Ashfaq-Khan M, Zevallos V. Non-celiac wheat sensitivity: differential diagnosis, triggers and implications. Best Pract Res Clin Gastroenterol 2015;29:469-76.

49. Carroccio A, Rini G, Mansueto P. Non-celiac wheat sensitivity is a more appropriate label than non-celiac gluten sensitivity. Gastroenterology 2014;146:320-1.
50. Catassi C, Alaedini A, Bojarski C, et al. The overlapping area of non-celiac gluten sensitivity (NCGS) and wheatsensitive irritable bowel syndrome (IBS): an update. Nutrients 2017;911:pii:E1268.

51. Gibson PR, Shepherd SJ. Personal view: food for thought-western lifestyle and susceptibility to Crohn's disease. The FODMAP hypothesis. Aliment Pharmacol Ther 2005;21:1399-409.

52. Biesiekierski JR, Peters SL, Newnham ED, et al. No effects of gluten in patients with self-reported non-celiac gluten sensitivity after dietary reduction of fermentable, poorly absorbed, short-chain carbohydrates. Gastroenterology 2013;145:320-8.

53. Biesiekierski JR, Newnham ED, Irving PM, et al. Gluten causes gastrointestinal symptoms in subjects without celiac disease: a double-blind randomized placebocontrolled trial. Am J Gastroenterol 2011;106:508-14.

54. Skodje GI, Sarna VK, Minelle IH, et al. Fructan, rather than gluten, induces symptoms in patients with selfreported non-celiac gluten sensitivity. Gastroenterology 2018;154:529-39.

55. McIntosh K, Reed DE, Schneider T, et al. FODMAPs alter symptoms and the metabolome of patients with IBS: a randomised controlled trial. Gut 2017;66:1241-51.

56. Halmos EP, Power VA, Shepherd SJ et al. A diet low in FODMAPs reduces symptoms of irritable bowel syndrome. Gastroenterology 2014;146:67-75.

57. Gibson P, Shepherd S. Food choice as a key management strategy for functional gastrointestinal symptoms. Am J Gastroenterol 2012;107:657-66.

58. Mansueto P, Seidita A, D'Alcamo A, Carroccio A. Role of FODMAPs in patients with irritable bowel syndrome. Nutr Clin Pract 2015;30:665-82. 